\title{
Short communication: In vitro antimicrobial susceptibility of Mycoplasma agalactiae strains isolated from dairy goats
}

\author{
A. Paterna, A. Sánchez, ${ }^{1}$ A. Gómez-Martín, J. C. Corrales, C. De la Fe, A. Contreras, and J. Amores \\ Research Group on Ruminant Health, Animal Health Department, School of Veterinary Sciences, Regional Campus of International Excellence \\ "Campus Mare Nostrum," University of Murcia, 30071 Murcia, Spain
}

\begin{abstract}
This study examined the susceptibility to several antimicrobials of 28 isolates of Mycoplasma agalactiae obtained from goats in a region (southeastern Spain) where contagious agalactia is endemic. For each isolate, the minimum inhibitory concentration (MIC) against 12 antimicrobials of the quinolone, macrolide, aminoglycoside, and tetracycline families was determined. The antimicrobials with the lowest MIC were enrofloxacin, ciprofloxacin, tylosin, and doxycycline, all with $\mathrm{MIC}_{90}$ (concentration at which growth of $90 \%$ of the isolates is inhibited) $<1 \mu \mathrm{g} / \mathrm{mL}$. Norfloxacin (a quinolone) showed a wide MIC range $(0.1-12.8 \mu \mathrm{g} / \mathrm{mL})$, suggesting a resistance mechanism toward this antimicrobial that was not elicited by enrofloxacin or ciprofloxacin (the other quinolones tested). Erythromycin showed the highest $\mathrm{MIC}_{90}$ such that its use against Mycoplasma agalactiae is not recommended. Finally, Mycoplasma agalactiae isolates obtained from goat herds with clinical symptoms of contagious agalactia featured higher $\mathrm{MIC}_{90}$ and $\mathrm{MIC}_{50}$ (concentration at which growth of $50 \%$ of the isolates is inhibited) values for many of the antimicrobials compared with isolates from asymptomatic animals. The relationship between the extensive use of antimicrobials in herds with clinical contagious agalactia and variations in MIC requires further study. Key words: Mycoplasma agalactiae, antimicrobial, minimum inhibitory concentration, contagious agalactia
\end{abstract}

\section{Short Communication}

Mycoplasma agalactiae is the main causative agent of contagious agalactia (CA), a syndrome that affects small ruminants and causes mastitis, arthritis, keratoconjunctivitis, pneumonia, and abortion. Contagious agalactia is distributed worldwide although it was traditionally reported to affect animals in southern Europe

Received December 17, 2012.

Accepted July 31, 2013.

${ }^{1}$ Corresponding author: asanlope@um.es
(Bergonier et al., 1997). The most significant symptom in ruminants reared for dairy purposes is mammary gland infection, which may lead to the complete loss of milk production as well as loss of the mammary gland itself. The disease first presents as an explosive outbreak affecting most lactating females in a herd and compromising herd viability as a consequence of milk and animal losses. Moreover, in endemic areas, the infection tends to persist in a chronic form and it is possible to isolate Mycoplasma agalactiae from herds with or without recent history of CA; for example, from auricular carriers or bulk tank milk samples (BTM) from asymptomatic herds (Mercier et al., 2007; Contreras et al., 2008). In goat herds, a single clone of Mycoplasma mycoides ssp. capri has been described during a clinical outbreak of $\mathrm{CA}$, and different strains of M. mycoides ssp. capri have been isolated from asymptomatic auricular carriers and BTM of herds in the absence of a recent mycoplasmosis episode (Tardy et al., 2007). In a recent study, $M$. agalactiae was isolated from clinical mastitis specimens and BTM in herds without clinical signs of CA in an endemic area (Amores et al., 2012). Further, the presence of different strains of $M$. agalactiae of different epidemiological origins has been reported in dairy goat farms in an endemic CA area (De la Fe et al., 2012). Besides losses in milk production, classic CA outbreaks or chronic infection in a herd generates serious economic losses arising from the management procedures that need to be implemented to control the disease.

The control of CA is complex and mainly based on antimicrobial treatment and preventive handling procedures. Because mycoplasmas lack a cell wall, they are not susceptible to the $\beta$-lactam class of antimicrobials. In addition, the chronic nature of CA infection in endemic areas has given rise to uncontrolled antimicrobial treatment, which could lead to the appearance of resistant strains (Ayling et al., 2000). The antimicrobial drug families known to be effective against mycoplasmas are macrolides, fluoroquinolones, tetracyclines, and aminoglycosides (Hirsh, 2000), macrolides and quinolones being widely used in endemic areas. However, some resistance to macrolides and fluoroquinolones has been observed and attributed to several gene mutations 
(Furneri et al., 2001; Lysnyansky et al., 2009). The diversity of genetic populations of $M$. agalactiae isolates from goats detected in endemic areas and the wide use of antimicrobials could lead to inefficient control therapy.

Considering the different clinical and epidemiological aspects of endemic CA, this study was designed to determine MIC of 12 antimicrobials against $28 \mathrm{M}$. agalactiae isolates. The isolates used in this study form part of our laboratory culture collection and were grouped according to the available information (clinical signs and anatomic origin). Samples were cultured in modified Hayflick broth, a medium specific for mycoplasmas, and characteristic mycoplasma colonies were visualized on agar. Single colonies were subcultured and the species identified by biochemical tests (tetrazolium reduction and film and spot production) and by PCR targeting the polC gene (Marenda et al., 2005). For the present purpose, isolates were selected according to epidemiological data. Eighteen of the isolates were obtained during 3 reported CA outbreaks from goats showing classic symptoms (mastitis, arthritis, or eye symptoms): 9 of these were selected from cultures of mastitic specimens, 2 from cultures of conjunctival swabs, 2 from cultures of swabs taken of the external auditory canal (EAC) in animals with mastitis, 2 from cultures of joint fluid, and 3 from cultures of the BTM of each herd. The remaining $10 \mathrm{M}$. agalactiae isolates were obtained from herds with no prior clinical CA outbreak: 6 from the BTM of 6 different herds, 3 from the EAC of 3 animals in 3 different herds, and 1 from a semen sample from a single buck. The isolates obtained from the EAC and semen were obtained after $M$. agalactiae had been detected in the BTM of the herds. A reference M. agalactiae strain (PG2, NCTC 10123) was also included.

The concentration range of the antimicrobials to be tested was determined by taking into account the active ingredient of each: streptomycin and erythromycin (1-128 $\mu \mathrm{g} / \mathrm{mL})$; doxycycline, ciprofloxacin, enrofloxacin, and tylosin $(0.006-0.8 \mu \mathrm{g} / \mathrm{mL})$; gentamicin, norfloxacin, spiramycin, kanamycin, spectinomycin, and neomycin $(0.1-12.8 \mu \mathrm{g} / \mathrm{mL})$. The MIC for each isolate and antimicrobial was determined according to the recommendations of Hannan (2000). In brief, a mycoplasma growth curve was prepared for each isolate. Cultures were grown in modified Hayflick medium supplemented with $1 \%$ phenol red in 96 -well round-bottomed plates. The antimicrobials were added to achieve each of the pre-established final concentrations and a final concentration of the mycoplasma cultures of $10^{5}$ to $10^{3}$ color-changing units $/ \mathrm{mL}$. We also prepared positive controls lacking antibiotics and negative controls lacking mycoplasma isolates. The plates were then sealed and incubated at $37^{\circ} \mathrm{C}$ until the positive control (antibiotic-free) changed color from red to yellow and the negative control (mycoplasma-free) remained red. The MIC was defined as the lowest concentration at which no bacterial growth (no color change) was observed. Depending on the isolate, the color change occurred after 24 to $72 \mathrm{~h}$ of incubation. For each group of isolates (from herds with clinical CA or asymptomatic herds), we determined the MIC range, $\mathrm{MIC}_{50}$, and $\mathrm{MIC}_{90}$ (the lowest concentration of antimicrobial at which growth of $50 \%$ or $90 \%$ of the isolates, respectively, is inhibited; Table 1).

Among the antimicrobials tested, enrofloxacin, ciprofloxacin, tylosin, and doxycycline showed the lowest MIC values, all with $\mathrm{MIC}_{90}<1 \mu \mathrm{g} / \mathrm{mL}$. Enrofloxacin had the lowest values $\left(\mathrm{MIC}_{90}=0.1 \mu \mathrm{g} / \mathrm{mL}\right)$ for both groups of isolates. In contrast, erythromycin had the highest $\mathrm{MIC}_{90}$ for both groups, in agreement with previous reports (Antunes et al., 2008; de Garnica et al., 2013). High MIC of erythromycin has also been described for Mycoplasma bovis and Mycoplasma hominis (Furneri et al., 2001; Francoz et al., 2005). These mycoplasmas are closely related to $M$. agalactiae and also belong to the hominis group. In effect, M. hominis is naturally resistant to erythromycin because of a mutation in the central loop of $23 \mathrm{~S}$ rRNA domain $\mathrm{V}$. This mutation is also present in Mycoplasma flocculare and Mycoplasma hyopneumoniae (Furneri et al., 2001). These 2 mycoplasmas also belong to the hominis group, suggesting that the resistance to erythromycin observed in $M$. agalactiae could be natural resistance common to the entire hominis group. This should be considered when treating CA, because erythromycin is used to treat mammary infections but is inefficient against $M$. agalactiae. The following breakpoints have been described for the effectiveness of tylosin against $M$. agalactiae: $\leq 1 \mu \mathrm{g} / \mathrm{mL}$ susceptible, $\leq 2 \mu \mathrm{g} / \mathrm{mL}$ intermediately susceptible, and $\geq 4 \mu \mathrm{g} / \mathrm{mL}$ resistant (Hannan, 2000). In our study, no $M$. agalactiae isolate showed a MIC $>4 \mu \mathrm{g} / \mathrm{mL}$, indicating their susceptibility to this antimicrobial, in contrast to the results obtained for the other macrolides.

Most of the aminoglycosides examined showed high $\mathrm{MIC}_{90}$, with streptomycin having the highest values $\left(\mathrm{MIC}_{90}\right.$ of $\left.32 \mu \mathrm{g} / \mathrm{mL}\right)$. It has been reported that the presence of thymidine at position 912 (Escherichia coli numbering) of the $16 \mathrm{~S}$ rRNA gene confers $E$. coli resistance to streptomycin (Cundliffe, 1990). This mutation is also present in mycoplasmas of the hominis group, in which $M$. agalactiae is included, and has been related to $M$. agalactiae resistance to streptomycin (Königsson et al., 2002).

Quinolones had lowest MIC values of antimicrobials against our $M$. agalactiae isolates, in accordance with 
Table 1. Minimum inhibitory concentrations ${ }^{1}(\mu \mathrm{g} / \mathrm{mL})$ of antimicrobials recorded for Mycoplasma agalactiae isolates obtained from dairy goat herds with or without clinical signs of contagious agalactia (CA)

\begin{tabular}{|c|c|c|c|c|c|c|c|}
\hline \multirow[b]{2}{*}{ Antimicrobial agent } & \multicolumn{3}{|c|}{$\begin{array}{l}\text { Isolates from herds with } \\
\text { clinical signs of CA }(\mathrm{n}=18)\end{array}$} & \multicolumn{3}{|c|}{$\begin{array}{l}\text { Isolates from herds without } \\
\text { clinical signs of CA }(\mathrm{n}=10)\end{array}$} & \multirow{2}{*}{$\begin{array}{c}\mathrm{PG} 2 \\
\mathrm{MIC}\end{array}$} \\
\hline & MIC range & $\mathrm{MIC}_{50}$ & $\mathrm{MIC}_{90}$ & MIC range & $\mathrm{MIC}_{50}$ & $\mathrm{MIC}_{90}$ & \\
\hline \multicolumn{8}{|l|}{ Quinolones } \\
\hline Norfloxacin & $0.1-12.8$ & 3.2 & 12.8 & $0.4-6.4$ & 1.6 & 6.4 & 0.8 \\
\hline Ciprofloxacin & $0.012-0.4$ & 0.2 & 0.4 & $0.012-0.4$ & 0.1 & 0.4 & 0.05 \\
\hline Enrofloxacin & $0.025-0.8$ & 0.05 & 0.1 & $0.012-0.8$ & 0.1 & 0.1 & 0.012 \\
\hline Tylosin & $0.05-0.8$ & 0.4 & 0.8 & $0.025-0.8$ & 0.4 & 0.8 & 0.05 \\
\hline Erythromycin & $16-128$ & 64 & 128 & $32-128$ & 64 & 128 & 16 \\
\hline \multicolumn{8}{|l|}{ Aminoglycosides } \\
\hline Neomycin & $0.2-12.8$ & 6.4 & 12.8 & $3.2-12.8$ & 6.4 & 12.8 & 0.8 \\
\hline Kanamycin & $0.8-12.8$ & 6.4 & 12.8 & $1.6-12.8$ & 3.2 & 6.4 & 0.8 \\
\hline Spectinomycin & $0.4-12.8$ & 3.2 & 6.4 & $0.8-6.4$ & 1.6 & 6.4 & 0.8 \\
\hline Gentamicin & $0.8-6.4$ & 1.6 & 6.4 & $0.2-3.2$ & 0.8 & 3.2 & 0.2 \\
\hline
\end{tabular}

${ }^{1} \mathrm{MIC}_{50}$ and $\mathrm{MIC}_{90}=$ lowest concentration at which growth of $50 \%$ and $90 \%$ of the isolates, respectively, is inhibited; PG2 = reference strain of Mycoplasma agalactiae (NCTC 10123).

in vitro susceptibilities reported for isolates from sheep (de Garnica et al., 2013). Enrofloxacin and ciprofloxacin showed the lowest $\mathrm{MIC}_{90}$ for both groups of isolates and the MIC range was consistently $<1 \mu \mathrm{g} / \mathrm{mL}$. Hannan (2000) proposed a MIC breakpoint for enrofloxacin of $\leq 0.5 \mu \mathrm{g} / \mathrm{mL}$ to define susceptible isolates, $\leq 1 \mu \mathrm{g} /$ $\mathrm{mL}$ for intermediately resistant isolates, and $\geq 2 \mu \mathrm{g} / \mathrm{mL}$ for resistant isolates. According to these breakpoints, 21 of the present isolates were resistant and 4 were intermediately resistant to norfloxacin. However, no isolate was resistant to ciprofloxacin and only 2 isolates showed intermediate resistance to enrofloxacin. The 3 quinolones tested here inhibit DNA gyrase or topoisomerase IV, blocking cell division. Quinolone resistance can arise because of point mutations that lead to amino acid substitutions in the quinolone resistancedetermining region of DNA gyrase subunits gyrA and gyr $\mathrm{B}$ or topoisomerase IV subunits $\operatorname{par} \mathrm{C}$ and $\operatorname{par} \mathrm{E}$ (Lysnyansky et al., 2009). The different susceptibilities to ciprofloxacin, enrofloxacin, and norfloxacin detected here are in contrast to the findings of a study in which enrofloxacin and difloxacin were observed to show similar MIC patterns and the same $\mathrm{MIC}_{50}$ for Mycoplasma gallisepticum and Mycoplasma synoviae (Gerchman et al., 2008). Those authors attributed the latter to cross resistance in this family of antimicrobials (Gerchman et al., 2008). However, in a more recent study, a discrepancy was noted between the MIC of enrofloxacin and quinolone resistance-determining region mutations in M. gallisepticum (Lysnyansky et al., 2012). Thus, the difference detected in the susceptibility of our isolates to norfloxacin compared with the other quinolones along with this recent discrepancy suggest that determining resistance to quinolones in mycoplasmas should not be based solely on the genes $\operatorname{par} C$ and gyrA. Other quinolone resistance mechanisms have been described in other bacteria (Hawkey, 2003) and further work is needed to establish whether these mechanisms also play a role in mycoplasmas.

The isolates from herds with clinical signs of CA had higher $\mathrm{MIC}_{90}$ and $\mathrm{MIC}_{50}$ for norfloxacin, kanamycin, streptomycin, and gentamicin than isolates from herds without clinical signs of disease (Table 1). Spectinomycin and spiramycin also showed higher $\mathrm{MIC}_{50}$ in isolates from herds with clinical CA signs, suggesting lower susceptibility of these isolates to some antimicrobials. This finding could be related to the intense use of antimicrobials in these herds, likely inducing mycoplasmal resistance (Ayling et al., 2000), and should be confirmed in further studies. Despite the fact that most of the antimicrobials analyzed are not licensed in Spain to treat mastitis in goats, the majority are, in fact, licensed for veterinary use. In the absence of a specific product for goats, veterinarians can use them to control caprine mastitis by the intramammary (erythromycin) or intramuscular route. Future studies should address whether the genetic diversity seen in the geographical area of study (De la Fe et al., 2012) is related to antimicrobial susceptibility.

Our findings indicate the natural resistance of $M$. agalactiae to erythromycin and streptomycin, such that these antimicrobials are not recommended for treatment of $M$. agalactiae infections. Quinolones had the lowest MIC values against M. agalactiae, although 
differences in $\mathrm{MIC}_{50}$ and $\mathrm{MIC}_{90}$ observed among the antimicrobials of this family suggest a different resistance mechanism in $M$. agalactiae than is reported for other mycoplasmas. Finally, M. agalactiae isolates from herds with clinical signs of CA showed higher MIC compared with isolates from herds with no symptoms. The relationship between MIC values and use of antimicrobials in an endemic area requires further study.

\section{ACKNOWLEDGMENTS}

This study was funded by project AGL2009-09128 awarded by the Spanish Ministry of Science and Innovation, Government of Spain. The authors thank Silvia Porras Pérez and Daniel Riquelme Moreno (technical personnel of the Veterinary Faculty, University of Murcia, Murcia, Spain). None of the authors has any financial or personal relationships that could inappropriately influence or bias the contents of this paper.

\section{REFERENCES}

Amores, J., A. Sánchez, A. Gómez-Martín, J. C. Corrales, A. Contreras, and C. De la Fe. 2012. Surveillance of Mycoplasma agalactiae and Mycoplasma mycoides ssp. capri in dairy goat herds. Small Rumin. Res. 102:89-93.

Antunes, N. T., M. M. Tavío, P. Assunção, R. S. Rosales, C. Poveda, C. De la Fe, M. C. Gil, and J. B. Poveda. 2008. In vitro susceptibilities of field isolates of Mycoplasma agalactiae. Vet. J. 177:436-438.

Ayling, R. D., S. E. Baker, M. L. Peek, A. J. Simon, and R. A. J. Nicholas. 2000. Comparison of in vitro activity of danofloxacin, florfenicol, oxytetracycline, spectinomycin and tilmicosin against recent field isolates of Mycoplasma bovis. Vet. Rec. 146:745-747.

Bergonier, D., X. Berthelot, and F. Poumarat. 1997. Contagious agalactia of small ruminants: Current knowledge concerning epidemiology, diagnosis and control. Rev. Sci. Tech. 16:848-873.

Contreras, A., R. E. Miranda, A. Sánchez, C. De la Fe, D. Sierra, C. Luengo, and J. C. Corrales. 2008. Presence of Mycoplasma species and somatic cell counts in bulk tank goats milk. Small Rumin. Res. 75:247-251.

Cundliffe, E. 1990. Recognition sites for antibiotics within rRNA. Pages 479-490 in The Ribosome: Structure, Function, and Evolution. W. E. Hill, A. Dahlberg, R. A. Garret, P. B. Moore, D. Schlessinger, and J. R. Warner, ed. American Society for Microbiology, Washington, DC

de Garnica, M. L., R. S. Rosales, C. Gonzalo, J. A. Santos, and R. A. J. Nicholas. 2013. Isolation, molecular characterization and anti- microbial susceptibilities of isolates of Mycoplasma agalactiae from bulk tank milk in an endemic area of Spain. J. Appl. Microbiol. 114:1575-1581.

De la Fe, C., J. Amores, F. Tardy, E. Sagne, L. X. Nouvell, and C. Citti. 2012. Unexpected genetic diversity of Mycoplasma agalactiae caprine isolates from an endemic geographically restricted area of Spain. BMC Vet. Res. 8:146. http://dx.doi.org/10.1186/17466148-8-146.

Francoz, D., M. Fortin, G. Fecteau, and S. Messier. 2005. Determination of Mycoplasma bovis susceptibilities against six antimicrobial agents using the E test method. Vet. Microbiol. 105:57-64.

Furneri, P. M., G. Rappazzo, M. P. Musumarra, P. Di Pietro, L. S. Catania, and L. S. Roccasalva. 2001. Two new point mutations at A2062 associated with resistance to 16-membered macrolide antibiotics in mutant strains of Mycoplasma hominis. Antimicrob. Agents Chemother. 45:2958-2060.

Gerchman, I., I. Lysnyansky, S. Perk, and S. Levishon. 2008. In vitro susceptibilities to fluoroquinolones in current and archived $\mathrm{Myco-}$ plasma gallisepticum and Mycoplasma synoviae isolates from meattype turkeys. Vet. Microbiol. 131:266-276.

Hannan, P. C. T. 2000. Guidelines and recommendations for antimicrobial minimum inhibitory concentration (MIC) testing against veterinary mycoplasma species. Vet. Res. 31:373-395.

Hawkey, P. M. 2003. Mechanism of quinolone action and microbial response. J. Antimicrob. Chemother. 51:29-35.

Hirsh, D. C. 2000. Mycoplasmas. Pages 466-468 in Antimicrobial Therapy in Veterinary Medicine. J. F. Prescott, J. D. Baggot, and R. D. Walker, ed. Iowa State University Press, Ames.

Königsson, M. H., G. Bölske, and K. E. Johansson. 2002. Intraspecific variation in the $16 \mathrm{~S}$ rRNA gene sequences of Mycoplasma agalactiae and Mycoplasma bovis strains. Vet. Microbiol. 85:209-220.

Lysnyansky, I., I. Gerchman, S. Levishon, I. Mikula, A. Faberwee, N. M. Ferguson, A. H. Noormohammadi, J. Spergser, and H. M. Windsor. 2012. Discrepancy between minimal inhibitory concentration to enrofloxacin and mutations present in the quinoloneresistance determining regions of Mycoplasma gallisepticum field strains. Vet. Microbiol. 160:222-226.

Lysnyansky, I., I. Mikula, I. Gerchman, and S. Levishon. 2009. Rapid detection of a point mutation in the $\operatorname{par} C$ gene associated with decreased susceptibility to fluoroquinolones in Mycoplasma bovis. Antimicrob. Agents Chemother. 53:4911-4914.

Marenda, M. S., E. Sagne, F. Poumarat, and C. Citti. 2005. Suppression subtractive hybridization as a basis to assess Mycoplasma agalactiae and Mycoplasma bovis genomic diversity and speciesspecific sequences. Microbiology 151:475-489.

Mercier, P., M. P. Pellet, E. Morignat, D. Calavas, and F. Poumarat. 2007. Prevalence of mycoplasmas in external ear canal of goats: Influence of the sanitary status of the herd. Small Rumin. Res. $73: 296-299$.

Tardy, F., P. Mercier, M. Solsona, E. Saras, and F. Poumarat. 2007. Mycoplasma mycoides ssp. mycoides biotype large colony isolates from healthy and diseased goats: Prevalence and typing. Vet. Microbiol. 121:268-277. 